\title{
Associations between gut microbiota and hand grip strength: a polygenetic scoring analysis and genome-wide environmental interaction study
}

Li Liu

Xi'an Jiaotong University https://orcid.org/0000-0003-0527-9250

Bolun Cheng

Xian Jiaotong University: Xi'an Jiaotong University

Yan Wen

Xian Jiaotong University: Xi'an Jiaotong University

Yumeng Jia

Xian Jiaotong University: Xi'an Jiaotong University

Shiqiang Cheng

Xian Jiaotong University: Xi'an Jiaotong University

Chujun Liang

Xian Jiaotong University: Xi'an Jiaotong University

Xiaomeng Chu

Xian Jiaotong University: Xi'an Jiaotong University

Jing Ye

Xian Jiaotong University: Xi'an Jiaotong University

Yao Yao

Xian Jiaotong University: Xi'an Jiaotong University

Xiaoxia Dai

Xian Jiaotong University: Xi'an Jiaotong University

Xiong Guo

Xian Jiaotong University: Xi'an Jiaotong University

Feng Zhang ( $\nabla$ fzhxjtu@mail.xjtu.edu.cn )

School of Public Health, Xi'an Jiaotong University Health Science Center https://orcid.org/0000-0003-1339-5956

\section{Research article}

Keywords: hand grip strength, gut microbiota, polygenetic risk score, Genome-wide by environment interaction study

Posted Date: November 30th, 2020

DOI: https://doi.org/10.21203/rs.3.rs-113419/v1

License: (a) (i) This work is licensed under a Creative Commons Attribution 4.0 International License. Read Full License 


\section{Abstract \\ Background}

We aim to explore the genetic association between hand grip strength (HGS) and gut microbiota (GM).

\section{Methods}

The genome-wide association study (GWAS) data of GM was obtained from a recently published study, involving 2,646 individuals. Phenotypic data of left HGS $(n=375,229)$ and right HGS $(n=375,279)$ were derived from the UK Biobank. Firstly, PLINK 2.0 was used to calculate $114 \mathrm{GM}$ related polygenetic risk score (PRS) of each subject based on GWAS result. Regression analysis was then conducted to evaluate the possible association of GM-PRS and HGS. Then genome-wide by environment interaction study (GWEIS) of GM related traits was then performed using a regression model adjusted for age, gender, $\mathrm{BMI}$ and 10 principal components.

\section{Results}

For PRS analysis, 13 candidate GM were identified to be associated with left HGS $(P<0.05)$, including G_Bifidobacterium $(P=8.36 \times$ $\left.10^{-4}\right), G$ _Clostridium_sensu_stricto $\left(P=2.69 \times 10^{-3}\right)$, and 10 significant $\mathrm{GM}$ for right HGS, such as $G$ _Acidaminococcus $(P=1.29 \times$ $\left.10^{-2}\right), G$ Streptococcus $\left(P=1.79 \times 10^{-2}\right)$. In addition, $4 \mathrm{GM}$ were found to be overlapped in association with both left and right HGS, including G_Bifidobacterium ( $\left.P_{\text {right HGS }}=2.78 \times 10^{-2}, P_{\text {left HGS }}=8.36 \times 10^{-4}\right)$. Furthermore, several genome-wide significant GWEIS associations were detected for HGS, including 3 significant SNPs interacted with $G$ _Butyricicoccus $\left(P<5 \times 10^{-8}\right)$ for right HGS (rs41310432, $P=3.86 \times 10^{-8}$; closest gene: NASP, rs1053941, $P=4.71 \times 10^{-8}$; closest gene: NASP, rs6671239, $P=4.73 \times 10^{-8}$, closest gene: GPBP1L1), and 1 suggestive intronic SNP in CACNA1H on chromosome 16 interacted with G_Butyricicoccus (rs139206507, $P=8.68 \times 10^{-8}$ ) for left HGS.

\section{Conclusions}

Our study holds great potential gaining a better understanding of the relationship between GM and HGS.

\section{Background}

Reduced muscle strength, as measured by hand grip strength (HGS), is considered a widely and accessible used proxy of muscle function. Therefore, grip strength serves as an established, simple and non-invasive marker of muscle fitness. Meanwhile, HGS has been used as a significant predictor and biomarker of overall health of individual. In addition, various studies have shown a close correlation between HGS and many diseases related conditions, including long-term morbidity and mortality in adults[1], bone mineral density[2, 3], fracture[4], depression[5], nutritional status[6], all-cause and cardiovascular mortality[7, 8]. Previous literatures showed environmental exposures contributed a lot to the development of HGS, including age, gender, body size, low physical activity and smoking [9]. The heritability of HGS was demonstrated to be approximately $30 \%-50 \%[10]$. Furthermore, multiple loci implicated in the genetic mechanisms of HGS have been found. Furthermore, Willems and colleagues identified 16 genome-wide significant loci associated with grip strength. And a number of these loci contain genes implicated in structure and function of skeletal muscle fibres (ACTG1)[11].

Trillions of cells make up the gut microbiota (GM), including bacteria, viruses, and fungi, and they play a fundamental role in maintaining our health. The importance of GM in host physiology has received a great deal of attention in the last decade. According to published studies, changes in gut microbial diversity and composition have been demonstrated to be associated with several diseases and conditions[12], including neurologic conditions and psychiatric disorders[13], inflammation and immune function[14], metabolic diseases[15], skeletal and muscle function[16]. For example, a review study presented that gut microbes may contribute to the development of obesity and other associated chronic metabolic conditions[17]. Intriguingly, it was also found that GM influences the skeletal homeostasis via affecting the host metabolism, immune function, hormone secretion, and the gut-brain axis, thus clarifying the relationship between GM and bone homeostasis[18]. 
Weaker muscle mass has been associated with reduced levels of physical performance[19]. In addition, reduced muscle mass and HGS reflected impaired nutritional status[20]. GM can influence the host physiology by regulating multiple processes, but few studies have assessed the possible association between GM composition and muscle mass. Accumulating evidence has indicated that reduced muscle mass was associated with GM composition in mice[21]. For example, Lahiri et al. demonstrated the existence of a gut microbiota-skeletal muscle axis in germ-free mice, suggesting a role for GM in regulating skeletal muscle mass and function [22]. Nevertheless, limited evidence has targeted the association between GM and muscle mass in human studies. Given the reliable and accuracy of HGS as an indicator of general health and nutritional status, while the important role of GM in host physiology, we assumed there might be some functional interactions between GM and muscle strength in human.

Recent years, genome-wide association studies (GWAS) have showed that common complex disorders have a polygenic genetic architecture, which can be combined into a polygenic risk score that predicts an individual's susceptibility to diseases. Therefore, based on GWAS results, a powerful new method for exploring the genetic structure of complex traits and predicting disease risk is proposed, polygenic risk score (PRS) analysis [23]. PRSs are an estimate of disease risk conducted by the individual based on the risk alleles and the corresponding effect sizes obtained from the GWAS summary statistics. Interestingly, it can determine whether the pleiotropic effects of variations found in one disease lead to an increased risk of the other. Nowadays, PRS analysis has been widely applied for detecting shared genetic aetiology among traits [24].

Usually, the pathogenesis of complex diseases is thought to involve the interaction of genetic factors and environmental exposures, which makes it difficult to study separately[25]. Genome-wide by environment interaction study (GWEIS) is a simple but powerful method taking into account of the gene $\times$ environmental interaction $(\mathrm{G} \times \mathrm{E})$ in the context of GWAS[26]. In addition, it is a complementation for GWAS analysis, for GWEIS focuses not only main effects, but also for marginal genetic effects, such as heterogeneity SNPs between subgroups defined by some environmental exposure[27]. G × E studies for common complex phenotypes are needed and may be informative for identifying novel genomic loci and providing better insight into mechanisms for complex diseases. Nowadays, GWEIS has been used as a powerful tool to uncover newly susceptibility loci for complex diseases. For example, a study suggested the APOE gene could be a possible candidate gene for the $\mathrm{G} \times \mathrm{E}$ interaction on grip strength trajectories [28].

In this study, using the GWAS datasets of GM and genotyping data of HGS from UK biobank, we firstly calculate the PRS of 114 GM related traits, and assessing the genetic correlation between GM related PRS and HGS. Then genome-wide gene $\times$ GM interaction analyses was performed to study the effect of GM on HGS. We hope that our study results provide novel clues for understanding the biological mechanism underlying the relationship between GM and HGS.

\section{Materials And Methods Ethic Statement}

The signed consents were provided in the participants visit assessment. Ethical approval of UK Biobank was granted by the National Health Service National Research Ethics Service (reference 11/NW/0382).

\section{Phenotype definition of hand grip strength}

Phenotypic data of HGS in this study was derived from the UK Biobank (https://www.ukbiobank.ac.uk/), which is a prospective study cohort consists of approximately 500,000 individuals aged between 49 and 60 years old[29]. Grip strength was assessed using a hydraulic hand dynamometer (Jamar J00105, Lafayette Instrument Company, Lafayette, Indiana), and the mean values of the right-hand and left-hand used in the analyses were expressed as kg per kg body weight [30].

\section{Genotyping, imputation and quality control (QC)}

Genotyping, imputation and QC were performed by the UK Biobank[29]. Briefly, SNP genotyping was performed using the Applied Biosystems UK BiLEVE Axiom Array by Affymetrix. Imputation was carried out with the IMPUTE4 program. The merged UK10K, 1000 Genomes phase 3 reference panels and the Haplotype Reference Consortium (HRC) data were used as the imputation reference panel, of which HRC data was defined as the main imputation reference panel[31]. For the sample-based QC, the metrics of missing rate and heterozygosity were used to identify the poor-quality samples. For marker-based QC, batch effects, plate effects, departures from Hardy-Weinberg equilibrium, sex effects, array effects, and discordance across control replicates, these are all tested 
statistically here to identify poor quality markers, checking for consistency of genotype calling across experimental factors. The detailed information about the DNA extraction and genotyping are described in a published study[31].

\section{GWAS data of gut microbiota}

The GWAS data of $114 \mathrm{GM}$ related traits was derived from a recently published study[32]. A total of 2,646 individuals from the Flanders region of Belgium were recruited into the Flemish Gut Flora Project (FGFP) and 2,688 stool samples were collected. Informed consent was provided by volunteers using mail. DNA was extracted from frozen faecal samples using the PowerMicrobiome RNA Isolation Kit (MOBIO Laboratories). For microbial traits, a presence/absence (P/A) phenotype and a zerotruncated (all zero values set as missing) abundance ( $\mathrm{AB}$ ) phenotype were generated. RNT (rank-normal transformation) and HB (hurdle binary) are two microbial trait model for continuous (RNT | AB) and binary (P/A) traits[32].

Genotyping was conducted using the Human Core Exome v.1.0 array and the Human Core Exome v.1.1 array. Totally, 509,886 variants and 2,293 individuals were remained after marker-based QC and sample-based QC. FGFP genotype data were phased using SHAPEIT3 and imputed with IMPUTE4 using UK10K and all 1000 Genome Project phase 3 samples as the reference pane. Detailed information about study recruitment and sample collection, sequencing and microbiome data processing, genotyping, QC and imputation were described in the published study[32].

\section{Polygenetic Risk Score analysis}

In this study, PLINK 2.0 was used to calculate polygenetic risk GM scores of each study subject based on GWAS result (http://www.cog-genomics.org/plink/2.0/)[33]. In brief, PRSs are the estimation of diseases risk, calculated by summing risk alleles, which are weighted by effect sizes derived from GWAS results. The GWAS summary statistics will be referred as the base and the dataset to be evaluated as the target. PRS analysis uses the beta coefficients from the GWAS as weight for each SNP allele in order to calculate an overall risk score for each individual subject in an independent target sample. Linear regression models were then used to test the association between the GM related PRS (predictor variable) and HGS variables (independent variables) using R (https://www.r-project.org/).

\section{Genome-wide environmental interaction study (GWEIS)}

Genome-wide gene-PRSs of GM interaction association analysis was performed here using a regression model adjusted for age, gender and principal components. PLINK 2.0 was used for the interaction analysis of the GWAS data (http://www.coggenomics.org/plink/2.0/)[33]. We used the following criteria for selecting SNPs. \. SNPs with call rates $₫ 0.9$ \. Hardy-Weinberg equilibrium exact test $P$ values $₫ 0.001 \rrbracket$. minor allele frequencies (MAFs) $₫ 0.01$. In addition, The unrelated subjects were generated with KING software, a rapid algorithm for relationship inference that allows the presence of unknown population substructure[34]. The quantile-quantile plot of interaction P values and the Manhattan plot of - $\log 10 \mathrm{P}$ of interaction were generated using "CMplot" package (https://github.com/YinLiLin/R-CMplot) in R platform. Significant SNPs for G $\times$ E effects were identified at a genome-wide significance threshold of $P<5 \times 10^{-8}$.

\section{Results}

\section{Basic characteristics of study subject}

Population features are shown in Table 1. 375,229 and 375,279 participants were included in the observational analyses of left HGS and right HGS, respectively.

Table 1

Basic characteristics of study subject

\begin{tabular}{|llllllll|}
\hline Variables & $\mathbf{N}$ & HGS, $\mathbf{k g}$ & Sex, Male\% & Age, years & Weight, $\mathbf{K g}$ & Height, $\mathbf{c m}$ & BMl, kg/m2 \\
\hline Left HGS & 375229 & $29.76 \pm 11.33$ & $173740,46.3 \%$ & $56.98 \pm 7.94$ & $78.34 \pm 15.89$ & $170.31 \pm 9.43$ & $27.42 \pm 4.75$ \\
\hline Right HGS & 375279 & $31.93 \pm 11.28$ & $173752,46.3 \%$ & $56.98 \pm 7.94$ & $78.34 \pm 15.89$ & $170.31 \pm 9.43$ & $27.42 \pm 4.75$ \\
\hline
\end{tabular}

Genetic correlation of gut microbiota-PRS and hand grip strength 
As a result, there were a total of 19 significant GM were identified to be associated with HGS $(P<0.05)$. Among them, 13 candidate GM were associated with left HGS, including G_Bifidobacterium $\left(P=8.36 \times 10^{-4}\right), G_{-}$Clostridium_sensu_stricto $\left(P=2.69 \times 10^{-3}\right)$, G_Veillonella $\left(P=2.77 \times 10^{-3}\right)$ (Table 2). For right HGS, we found 10 significant associated $\mathrm{GM}$, such as G_Acidaminococcus $(P=$ $\left.1.29 \times 10^{-2}\right), G \_$Streptococcus $\left(P=1.79 \times 10^{-2}\right)$, O_Selenomonadales $\left(P=2.62 \times 10^{-2}\right)$ (Table 3$)$.

Table 2

Candidate gut microbiota associated with left hand grip strength

\begin{tabular}{|llll|}
\hline Gut Microbiota & Estimate & Std. Error & $P$ value \\
\hline${ }^{\text {a G_Bifidobacterium_RNT }}$ & 1.5541 & 0.4652 & $8.36 \times 10^{-4}$ \\
\hline${ }^{\text {a}}$ G_Clostridium_sensu_stricto_HB & -0.4085 & 0.1361 & $2.69 \times 10^{-3}$ \\
\hline G_Veillonella_RNT & 0.5078 & 0.1697 & $2.77 \times 10^{-3}$ \\
\hline G_Butyricicoccus_RNT & -1.0452 & 0.3935 & $7.91 \times 10^{-3}$ \\
\hline G_Acidaminococcus_RNT & -0.2957 & 0.1162 & $1.09 \times 10^{-2}$ \\
\hline aF_Enterobacteriaceae_HB & -0.3908 & 0.1566 & $1.26 \times 10^{-2}$ \\
\hline G_Ruminococcus_RNT & 1.0394 & 0.4722 & $2.77 \times 10^{-2}$ \\
\hline G_Escherichia_Shigella_RNT & -0.5108 & 0.2326 & $2.81 \times 10^{-2}$ \\
\hline G_Parasutterella_HB & 0.7199 & 0.3367 & $3.25 \times 10^{-2}$ \\
\hline ap_Firmicutes_RNT & 0.6521 & 0.3108 & $3.59 \times 10^{-2}$ \\
\hline G_unclassified_C_Clostridia_RNT & 0.4384 & 0.2176 & $4.39 \times 10^{-2}$ \\
\hline F_Veillonellaceae_RNT & 0.7234 & 0.3649 & $4.74 \times 10^{-2}$ \\
\hline aO_Lactobacillales_RNT & 0.4826 & 0.2439 & $4.79 \times 10^{-2}$ \\
\hline Note: a G, genus; F, family; P, phylum; O, order; RNT, rank-normal transformation; HB, hurdle binary. \\
\hline
\end{tabular}

After further comparison, $4 \mathrm{GM}$ were found to be overlapped in association with both left and right HGS, including G_Bifidobacterium $\left(P_{\text {right HGS }}=2.78 \times 10^{-2}, P_{\text {left HGS }}=8.36 \times 10^{-4}\right), G \_C l o s t r i d i u m \_s e n s u \_s t r i c t o\left(P_{\text {right HGS }}=9.72 \times 10^{-4}, P_{\text {left HGS }}=2.69 \times 10^{-3}\right)$, G_Butyricicoccus $\left(P_{\text {right HGS }}=3.26 \times 10^{-3}, P_{\text {left HGS }}=7.91 \times 10^{-3}\right)$, and $G \_$Acidaminococcus $\left(P_{\text {right HGS }}=1.29 \times 10^{-2}, P_{\text {left HGS }}=1.09 \times\right.$ $10^{-2}$ ). 


\begin{tabular}{|c|c|c|c|}
\hline Gut Microbiota & Estimate & Std. Error & P value \\
\hline${ }^{\mathrm{a}} \mathrm{G} \_$Clostridium_sensu_stricto_HB & -0.4493 & 0.1362 & $9.72 \times 10^{-4}$ \\
\hline 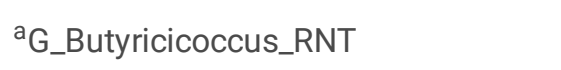 & -1.1588 & 0.3938 & $3.26 \times 10^{-3}$ \\
\hline G_Acidaminococcus_RNT & -0.2890 & 0.1162 & $1.29 \times 10^{-2}$ \\
\hline G_Streptococcus_HB & -1.6129 & 0.6809 & $1.79 \times 10^{-2}$ \\
\hline${ }^{\mathrm{a} O}$ _Selenomonadales_RNT & 0.6640 & 0.2986 & $2.62 \times 10^{-2}$ \\
\hline G_Bifidobacterium_RNT & 1.0238 & 0.4655 & $2.78 \times 10^{-2}$ \\
\hline G_unclassified_P_Bacteroidetes_HB & 0.2453 & 0.1165 & $3.52 \times 10^{-2}$ \\
\hline G_unclassified_P_Proteobacteria_HB & 0.4275 & 0.2051 & $3.71 \times 10^{-2}$ \\
\hline G_Collinsella_RNT & 0.9472 & 0.4734 & $4.54 \times 10^{-2}$ \\
\hline G_Victivallis_HB & -0.2366 & 0.1197 & $4.81 \times 10^{-2}$ \\
\hline
\end{tabular}

Note: ${ }^{\text {G }}$, genus; O, order; RNT, rank-normal transformation; HB, hurdle binary.

\section{GWEIS of hand grip strength}

Manhattan and quantile-quantile plots for all $P$ values of the gene-GM interactions are shown in Figs. 1. Several genome-wide significant GWEIS associations were detected for HGS in UK Biobank (Table 4). For right HGS, we found 3 significant SNPs interacted with G_Butyricicoccus $\left(P<5 \times 10^{-8}\right)$, including two significant SNPs on chromosome $1\left(\mathrm{rs} 41310432, P=3.86 \times 10^{-8}\right.$; rs1053941, $P=4.71 \times 10^{-8}$; closest gene: NASP), and an intronic SNP in GPBP1L1 on chromosome 1 (rs6671239, $P=4.73 \times 10^{-8}$ ). Also, there were 2 suggestive SNPs interacted with G_Butyricicoccus $\left(P<1 \times 10^{-7}\right.$ ), such as rs 11585275 (closest gene: GPBP1L1, $P=$ $5.10 \times 10^{-8}$ ), rs9429173(closest gene: IPP, $P=6.10 \times 10^{-8}$ ). For left HGS, we found 1 suggestive intronic SNP in CACNA1H on chromosome 16 interacted with G_Butyricicoccus (rs139206507, $\left.P=8.68 \times 10^{-8}\right)$. 
Table 4

Genome-wide significant GWEI associations detected for HGS

\begin{tabular}{|c|c|c|c|c|c|c|c|}
\hline Variables & Gut microbiota & $\begin{array}{l}\text { Overlapped } \\
\text { Gene }\end{array}$ & Variation ID & Chromosome & Position & Location & $\begin{array}{l}P \\
\text { value }\end{array}$ \\
\hline $\begin{array}{l}\text { Right } \\
\text { HGS }\end{array}$ & ${ }^{\mathrm{a}} G_{-}$Butyricicoccus_RNT & NASP & rs41310432 & 1 & 45618034 & $\begin{array}{l}\text { non-coding } \\
\text { intronic,intronic }\end{array}$ & $\begin{array}{l}3.86 \\
\times \\
10^{-8}\end{array}$ \\
\hline $\begin{array}{l}\text { Right } \\
\text { HGS }\end{array}$ & G_Butyricicoccus_RNT & NASP & rs1053941 & 1 & 45618711 & 3utr,3downstream & $\begin{array}{l}4.71 \\
\times \\
10^{-8}\end{array}$ \\
\hline $\begin{array}{l}\text { Right } \\
\text { HGS }\end{array}$ & G_Butyricicoccus_RNT & GPBP1L1 & rs6671239 & 1 & 45664441 & $\begin{array}{l}\text { non-coding } \\
\text { intronic,intronic }\end{array}$ & $\begin{array}{l}4.73 \\
x \\
10^{-8}\end{array}$ \\
\hline $\begin{array}{l}\text { Right } \\
\text { HGS }\end{array}$ & G_Butyricicoccus_RNT & GPBP1L1 & rs 11585275 & 1 & 45630317 & $\begin{array}{l}\text { non-coding } \\
\text { intronic,intronic }\end{array}$ & $\begin{array}{l}5.10 \\
\times \\
10^{-8}\end{array}$ \\
\hline $\begin{array}{l}\text { Right } \\
\text { HGS }\end{array}$ & G_Butyricicoccus_RNT & IPP & rs9429173 & 1 & 45697805 & $\begin{array}{l}\text { intronic, non- } \\
\text { coding intronic }\end{array}$ & $\begin{array}{l}6.10 \\
\times \\
10^{-8}\end{array}$ \\
\hline Left HGS & G_Butyricicoccus_RNT & CACNA1H & rs139206507 & 16 & 1189790 & intronic & $\begin{array}{l}8.68 \\
\times \\
10^{-8}\end{array}$ \\
\hline
\end{tabular}

\section{Discussion}

Despite evidence suggesting that altered GM has been implicated in the pathogenesis of several complicated diseases, little work has been done to explore the genetic association between GM and HGS. In this study, using PRS and GWEIS analysis, several genetic associations between GM and HGS were identified, as well as significant loci interacted with GM effecting HGS. Polygenic risk scores (PRSs) analyses incorporating $\mathrm{G} \times \mathrm{E}$ effects provided evidence of shared aetiologies between HGS and GM, which might improve efficiency for candidate gene discovery.

Bifidobacteria was found to be associated with both left and right HGS in this study. Bifidobacteria are a group of bacteria that normally live in the intestines, most of which can be considered as a probiotic and taken by mouth as medicine. The role of Bifidobacteria in muscle function has been well studied recently. An experimental study investigated the effects of Bifidobacterium breve B-3 (B-3) on muscle function on mice[35]. And it turned out that the administration of B-3 on the mouse model could increase muscle mass and affect muscle metabolism, and the grip strength of heat-killed B-3 group was significantly higher than that of the control group[35]. Similarity, another study suggested that dietary supplementation with Bifidobacterium breve BR03 attenuates performance decrements and muscle tension in the days following muscle-damaging exercise [36]. Furthermore, Ni et al. and colleagues found Lactobacillus and Bifidobacterium supplementation enhanced muscle strength and function in aged mice by the GM regulation [37]. Also, the protecting role of Bifidobacterium in against obesity has been proposed [38]. Taken altogether, findings from previous work we discussed above indicated the important role of Bifidobacterium in muscle mass and metabolism on mice. However, the number of evidences about this relationship in human studies remained scarce. In our analysis, we found Bifidobacteria was associated with both right and left HGS in human. Further supporting evidence is needed to prove our results.

G_Butyricicoccus is another common significant GM associated with both right and left HGS in our study. Interesting, the interaction between nutrients and the GM in the context of obesity and related metabolic diseases has been well studied [39].Among them, Butyricicoccus is one of the obesity related GM. For example, an experimental analysis found after transplantation of the GM from obese individuals to high-fat diet-fed mice, they responded differently to inulin supplementation, with Butyricimonas associated with the observed metabolic outcomes (decrease in adiposity and hepatic steatosis) in human-obesity mice[40]. Furthermore, Butyricicoccus drives the decrease of body mass index in response to inulin in obese individuals, suggesting the important role of 
characterizing the specific consortia of bacteria in the context of obesity and metabolic diseases when improving in metabolic disorders by inulin[40]. Studies showed the potential role of Butyricicoccus in exercise physiology. For example, Tung et al. found a greater abundance and diversity of GM in intrinsic high exercise capacity mice than in low exercise capacity mice, indicating the potential effect of GM and functional proteins on intrinsic exercise capacity[41]. The important role of nutrients in muscle strength and HGS has been revealed in previous study[42], which is consistent with our results.

O_Lactobacillales is found to be associated with left HGS in our analysis. Lactobacillales is a well-known probiotic among the ingested-microorganism probiotics. Various biological function of Lactobacillales has been explored, including treating obesity [43], enhancing muscle strength and function[37], improving insulin sensitivity[44]. A study conducted on mouse model found forelimb grip strengths were 1.31 and 1.40 fold higher in the Lactobacillus plantarum TWK10-1X and Lactobacillus plantarum TWK10-5X groups than in the vehicle treatment group, indicating long-term supplementation with Lactobacillus plantarum TWK10 might increase in muscle mass and strength [45]. Similar conclusion about the role of Lactobacillus plantarum TWK10 in physiological homeostasis and health promotion was also drawn in human study [46]. In addition, another study evaluated the effect of Lactobacillus rhamnosus on GM, changes in permeability, and insulin sensitivity and signaling in high-fat diet and control animals, and they suggested the important role of probiotics to prevent and treat patients with obesity and insulin resistance [47].

G_Veillonella, G_Ruminococcus and P_Firmicutes were also identified to be related with HGS in our study. An analysis found Ruminococcus and Veillonella were significantly related to muscle improvement outcomes after soy-whey blended protein (SWP) treatment in hematopoietic stem cell transplantation patients, suggesting that intestinal microbiota might affect the regulation of muscle metabolism [48]. Interesting, gender and body mass index may influence intestinal microbiota. It was found that Veillonella is significantly higher in fecal samples in men compared to women, and these differences may be influenced by the grade of obesity [49]. The Firmicutes is one of two groups of beneficial bacteria that dominant in the human gut. A meta-analysis concluded that changes in Firmicutes and Bacteroidetes phyla/species levels might be significant indicators for childhood obesity[50]. Likewise, same conclusion was also drawn by other literature [51]. A high value of evidence indicated that muscle mass is an important criterion in nutrition status diagnosis, and HGS is used as an efficient tool for evaluating muscle functioning. Given the fact that the important role of HGS as an outcome predictor and marker of nutritional status[52], the relationship among microbiota, HGS and obesity deserves to be deeply explored. Our results were consistent with previous studies.

For GWEIS analysis, several significant SNPs were identified to be interacted with G_Butyricicoccus for HGS. Among them, rs41310432 is the most significant one (NASP, $P=3.86 \times 10^{-8}$ ). Nuclear autoantigenic sperm protein (NASP) encodes a H1 histone binding protein and it is cell-cycle regulated [53]. It is expressed in all cells undergoing division. Functions of NASP mainly involve liver cancer [54], immune response [55], and cell proliferation [56]. For example, previous studies have shown that in lupus model mice, NASP gene mutation can change the proportion of immune cells in the spleen and aggravate the autoimmune response [55]. In addition, Yang and colleagues found somatic NASP negatively regulates TRAF6, and is a key regulator of innate immunity [57]. Interestingly, TRAF6 has been demonstrated to be a novel regulator of skeletal muscle atrophy [58]. Another study showed that overexpression of miR-125b-5p targeting TRAF6 may provide a promising therapeutic approach to treat muscle atrophy [59]. We found NASP is interacted with G_Butyricicoccus for HGS in our study. Given the potential role of inflammation [60] and muscle mass in HGS based on previous studies, the association between HGS and NASP needs to be studied further.

Another suggestive gene we identified in GWEIS analysis is CACNA1H. Calcium Voltage-Gated Channel Subunit Alpha1 H (CACNA1H), encodes a T-type member of the alpha-1 subunit family, a protein in the voltage-dependent calcium channel complex. Previous study indicated compound heterozygous CACNA1H mutations might lead to severe congenital amyotrophy [61]. Another experimental analysis suggested reduced expression of $\mathrm{CACNA} 1 \mathrm{H}$ related to multiple vaginal delivery is associated with muscular atrophy, indicating the key regulator role of CACNA1H in skeletal muscle function[62]. In our study, we found CACNA1H is interacted with G_Butyricicoccus and might affect HGS in UK Biobank samples. Further analyses are warranted to validate our results.

In this study, we analyzed the association between HGS and GM using large sample from UK Biobank via utilizing PRS and GWEIS analysis. PRSs analyses incorporating $\mathrm{G} \times \mathrm{E}$ effects provided evidence of shared aetiologies between HGS and GM. As far as we known, this is the first systemic study exploring the effect of GM as environmental factor on HGS. Besides, insight on interactions between GM and muscle mass from previous studies are mostly based on mouse studies rather than human studies. In addition, the large sample sizes guaranteed the accuracy of our results. Our study holds great potential for clarifying the functional relevance of GM with HGS. 
Several issues in our study should be noted. Although several significant GM were identified to share common genetic etiology with HGS, as well as significant genes interacted with GM for HGS, further biological research should determine if these genes are true susceptibility loci for HGS in relation to GM. In addition, participants from our study are all European ancestry and so the conclusions that can be drawn are limited. Further analysis should include other ethnic groups.

\section{Conclusions}

In summary, we identified genetic associations between GM and HGS using PRS analysis and significant genes interacted with GM effecting HGS using GWEIS analysis. Our results have provided entry points to analyze the mechanisms underlying the relationship between GM and HGS and providing an insight on the pathogenesis and therapeutic strategies of these musculoskeletal diseases.

\section{Abbreviations}

HGS

hand grip strength

GM

gut microbiota

GWAS

genome-wide association study

PRS

polygenetic risk score

GWEIS

genome-wide by environment interaction study

QC

quality control

HRC

Haplotype Reference Consortium

FGFP

Flemish Gut Flora Project

$\mathrm{P} / \mathrm{A}$

presence/absence

AB

abundance

RNT

rank-normal transformation

$\mathrm{HB}$

hurdle binary

MAFs

minor allele frequencies

\section{Declarations}

\section{Ethics approval and consent to participate}

This study was approved by the Institutional Review Board of Xi'an Jiaotong University. The signed consents were provided in the participants visit assessment. Ethical approval of UK Biobank was granted by the National Health Service National Research Ethics Service (reference 11/NW/0382).

\section{Consent for publication}

Not applicable 
Availability of data and materials

The datasets used and/or analysed during the current study are available from the corresponding author on reasonable request.

\section{Competing interests}

The authors declare that they have no competing interests.

\section{Funding}

This study was funded by the National Natural Scientific Foundation of China (81673112); the Key projects of international cooperation among governments in scientific and technological innovation (2016YFE0119100); the Natural Science Basic Research Plan in Shaanxi Province of China (2017JZ024); and the Fundamental Research Funds for the Central Universities.

\section{Authors' contributions}

Liu and Bolun Cheng drafted the manuscript. Zhang, Jia and Wen designed the study. Guo and Zhang provided the key datasets regarding our manuscript. Shiqiang Cheng, Liang, Ye and Yao performed the statistical analyses. Dai and Chu provided feasible advice on data analysis and drafting manuscript. All authors read and approved the final manuscript. All authors discussed the results and commented on the manuscript.

\section{Acknowledgements}

Thanks to all participants in this study.

\section{References}

1. Aihie Sayer, A. and T. Kirkwood, Grip strength and mortality: A biomarker of ageing? The Lancet, 2015. 386.

2. Kaya, A., S. Ozgocmen, O. Ardicoglu, A. Kamanli, and H. Gudul, Relationship between grip strength and hand bone mineral density in healthy adults. Arch Med Res, 2005. 36(5): p. 603-6.

3. Kritz-Silverstein, D., ., E. Barrett-Connor, . \%J Journal of Bone, M.R.t.O.J.o.t.A.S.f. Bone, and M. Research, Grip strength and bone mineral density in older women. 2010. 9(1): p. 45-51.

4. Denk, K., S. Lennon, S. Gordon, and R.L. Jaarsma, The association between decreased hand grip strength and hip fracture in older people: A systematic review. Exp Gerontol, 2018. 111: p. 1-9.

5. Ashdown-Franks, G., B. Stubbs, A. Koyanagi, F. Schuch, J. Firth, N. Veronese, et al., Handgrip strength and depression among 34,129 adults aged 50 years and older in six low- and middle-income countries. Journal of Affective Disorders, 2019. 243: p. 448-454.

6. Norman, K., N. Stobäus, M.C. Gonzalez, J.-D. Schulzke, and M. Pirlich, Hand grip strength: Outcome predictor and marker of nutritional status. Clinical Nutrition, 2011. 30(2): p. 135-142.

7. Leong, D.P., K.K. Teo, S. Rangarajan, P. Lopez-Jaramillo, A. Avezum, Jr., A. Orlandini, et al., Prognostic value of grip strength: findings from the Prospective Urban Rural Epidemiology (PURE) study. Lancet, 2015. 386(9990): p. 266-73.

8. Chainani, V., S. Shaharyar, K. Dave, V. Choksi, S. Ravindranathan, R. Hanno, et al., Objective measures of the frailty syndrome (hand grip strength and gait speed) and cardiovascular mortality: A systematic review. Int J Cardiol, 2016. 215: p. 487-93.

9. Ola, S., C.A. Reynolds, F. Deborah, E.B. Marie, N.L. Pedersen, A.A.K.J.A. Dahl, et al., Factors associated with grip strength decline in older adults. (2): p. 269-74.

10. Arden, N.K. and T.D. Spector, Genetic Influences on Muscle Strength, Lean Body Mass, and Bone Mineral Density: A Twin Study. 1997. 12(12): p. 2076-2081.

11. Willems, S.M., D.J. Wright, F.R. Day, K. Trajanoska, P.K. Joshi, J.A. Morris, et al., Large-scale GWAS identifies multiple loci for hand grip strength providing biological insights into muscular fitness. Nature Communications, 2017. 8(1): p. 16015. 
12. Jackson, M.A., S. Verdi, M.-E. Maxan, C.M. Shin, J. Zierer, R.C.E. Bowyer, et al., Gut microbiota associations with common diseases and prescription medications in a population-based cohort. Nature Communications, 2018. 9(1): p. 2655.

13. Rogers, G.B., D.J. Keating, R.L. Young, M.L. Wong, J. Licinio, and S. Wesselingh, From gut dysbiosis to altered brain function and mental illness: mechanisms and pathways. Mol Psychiatry, 2016. 21(6): p. 738-48.

14. Honda, K. and D.R. Littman, The microbiota in adaptive immune homeostasis and disease. Nature, 2016. 535(7610): p. 75-84.

15. Patterson, E., P.M. Ryan, J.F. Cryan, T.G. Dinan, R.P. Ross, G.F. Fitzgerald, et al., Gut microbiota, obesity and diabetes. Postgrad Med J, 2016. 92(1087): p. 286-300.

16. Lahiri, S., H. Kim, I. Garcia-Perez, M.M. Reza, K.A. Martin, P. Kundu, et al., The gut microbiota influences skeletal muscle mass and function in mice. Sci Transl Med, 2019. 11(502).

17. Abenavoli, L., E. Scarpellini, C. Colica, L. Boccuto, and R.J.N. Capasso, Gut Microbiota and Obesity: A Role for Probiotics. 2019. 11(11): p. 2690.

18. Behera, J., J. Ison, S.C. Tyagi, and N. Tyagi, The role of gut microbiota in bone homeostasis. Bone, 2020. 135 : p. 115317.

19. Bohannon, R.W., Muscle strength: clinical and prognostic value of hand-grip dynamometry. Curr Opin Clin Nutr Metab Care, 2015. 18(5): p. 465-70.

20. Neves, T., C.A. Fett, E. Ferriolli, M.G. Crespilho Souza, A.D. Dos Reis Filho, M.B. Martin Lopes, et al., Correlation between muscle mass, nutritional status and physical performance of elderly people. Osteoporosis and sarcopenia, 2018. 4(4): p. 145-149.

21. Ticinesi, A., A. Nouvenne, N. Cerundolo, P. Catania, B. Prati, C. Tana, et al., Gut Microbiota, Muscle Mass and Function in Aging: A Focus on Physical Frailty and Sarcopenia. Nutrients, 2019. 11(7): p. 1633.

22. Lahiri, S., H. Kim, I. Garcia-Perez, M.M. Reza, K.A. Martin, P. Kundu, et al., The gut microbiota influences skeletal muscle mass and function in mice. Science translational medicine, 2019. 11(502): p. eaan5662.

23. Lewis, C.M. and E. Vassos, Polygenic risk scores: from research tools to clinical instruments. Genome Medicine, 2020. 12(1): p. 44.

24. Ruisch, I.H., A. Dietrich, M. Klein, S.V. Faraone, J. Oosterlaan, J.K. Buitelaar, et al., Aggression based genome-wide, glutamatergic, dopaminergic and neuroendocrine polygenic risk scores predict callous-unemotional traits. Neuropsychopharmacology, 2020. 45(5): p. 761-769.

25. Klimek, P., S. Aichberger, and S. Thurner, Disentangling genetic and environmental risk factors for individual diseases from multiplex comorbidity networks. Scientific Reports, 2016. 6(1): p. 39658.

26. Milmont, C., J.P. Lewinger, and W. Gauderman, Gene-Environment Interaction in Genome-Wide Association Studies. American journal of epidemiology, 2008. 169: p. 219-26.

27. Thomas, D., Methods for investigating gene-environment interactions in candidate pathway and genome-wide association studies. Annu Rev Public Health, 2010. 31: p. 21-36.

28. Petersen, I., N.L. Pedersen, T. Rantanen, W.S. Kremen, W. Johnson, M.S. Panizzon, et al., GxE Interaction Influences Trajectories of Hand Grip Strength. Behavior Genetics, 2016. 46(1): p. 20-30.

29. Sudlow, C., J. Gallacher, N. Allen, V. Beral, P. Burton, J. Danesh, et al., UK Biobank: An Open Access Resource for Identifying the Causes of a Wide Range of Complex Diseases of Middle and Old Age. PLOS Medicine, 2015. 12(3): p. e1001779.

30. Spruit, M.A., M.J.H. Sillen, M.T.J. Groenen, E.F.M. Wouters, and F.M.E. Franssen, New Normative Values for Handgrip Strength: Results From the UK Biobank. Journal of the American Medical Directors Association, 2013. 14(10): p. 775.e5-775.e11.

31. Bycroft, C., C. Freeman, D. Petkova, G. Band, L.T. Elliott, K. Sharp, et al., The UK Biobank resource with deep phenotyping and genomic data. Nature, 2018. 562(7726): p. 203-209.

32. Hughes, D.A., R. Bacigalupe, J. Wang, M.C. Rühlemann, R.Y. Tito, G. Falony, et al., Genome-wide associations of human gut microbiome variation and implications for causal inference analyses. Nature Microbiology, 2020. 5(9): p. $1079-1087$.

33. Purcell, S., B. Neale, K. Todd-Brown, L. Thomas, M.A.R. Ferreira, D. Bender, et al., PLINK: a tool set for whole-genome association and population-based linkage analyses. American journal of human genetics, 2007. 81(3): p. 559-575.

34. Chen, W.M., Robust relationship inference in genome-wide association studies. Bioinformatics, 2010. 26(22): p. $2867-2873$.

35. Toda, K., Y. Yamauchi, A. Tanaka, T. Kuhara, T. Odamaki, S. Yoshimoto, et al., Heat-Killed Bifidobacterium breve B-3 Enhances Muscle Functions: Possible Involvement of Increases in Muscle Mass and Mitochondrial Biogenesis. Nutrients, 2020. 12(1): p.

Page 11/13 
219.

36. Jäger, R., M. Purpura, J.D. Stone, S.M. Turner, A.J. Anzalone, M.J. Eimerbrink, et al., Probiotic Streptococcus thermophilus FP4 and Bifidobacterium breve BRO3 Supplementation Attenuates Performance and Range-of-Motion Decrements Following Muscle Damaging Exercise. Nutrients, 2016. 8(10).

37. Ni, Y., X. Yang, L. Zheng, Z. Wang, L. Wu, J. Jiang, et al., Lactobacillus and Bifidobacterium Improves Physiological Function and Cognitive Ability in Aged Mice by the Regulation of Gut Microbiota. 2019. 63(22): p. e1900603.

38. Mazloom, K., I. Siddiqi, and M. Covasa, Probiotics: How Effective Are They in the Fight against Obesity? Nutrients, 2019. 11(2).

39. Sonnenburg, J.L. and F. Bäckhed, Diet-microbiota interactions as moderators of human metabolism. Nature, 2016. 535(7610): p. $56-64$.

40. Rodriguez, J., S. Hiel, and A.M. Neyrinck, Discovery of the gut microbial signature driving the efficacy of prebiotic intervention in obese patients. 2020. 69(11): p. 1975-1987.

41. Tung, Y.-T., Y.-J. Hsu, C.-C. Liao, S.-T. Ho, C.-C. Huang, and W.-C. Huang, Physiological and Biochemical Effects of Intrinsically High and Low Exercise Capacities Through Multiomics Approaches. Frontiers in physiology, 2019. 10: p. 1201-1201.

42. A, A.G., C.M.B.C. D, F.H. D, J.P. D, A.R. A, and S.R.G. B, Associations between diet and handgrip strength: a cross-sectional study from UK Biobank. Mechanisms of Ageing and Development. 189.

43. Mekkes, M.C., T.C. Weenen, R.J. Brummer, and E. Claassen, The development of probiotic treatment in obesity: a review. Benef Microbes, 2014. 5(1): p. 19-28.

44. Andreasen, A.S., N. Larsen, T. Pedersen-Skovsgaard, R.M. Berg, K. Møller, K.D. Svendsen, et al., Effects of Lactobacillus acidophilus NCFM on insulin sensitivity and the systemic inflammatory response in human subjects. Br J Nutr, 2010. 104(12): $\mathrm{p}$. 1831-8.

45. Chen, Y.-M., L. Wei, Y.-S. Chiu, Y.-J. Hsu, T.-Y. Tsai, M.-F. Wang, et al., Lactobacillus plantarum TWK10 Supplementation Improves Exercise Performance and Increases Muscle Mass in Mice. Nutrients, 2016. 8(4): p. 205-205.

46. Huang, W.C., Y.J. Hsu, H. Li, N.W. Kan, Y.M. Chen, J.S. Lin, et al., Effect of Lactobacillus Plantarum TWK10 on Improving Endurance Performance in Humans. Chin J Physiol, 2018. 61(3): p. 163-170.

47. Bagarolli, R.A., N. Tobar, A.G. Oliveira, T.G. Araújo, B.M. Carvalho, G.Z. Rocha, et al., Probiotics modulate gut microbiota and improve insulin sensitivity in DIO mice. J Nutr Biochem, 2017. 50: p. 16-25.

48. Ren, G., J. Zhang, M. Li, Z. Tang, Z. Yang, G. Cheng, et al., Gut microbiota composition influences outcomes of skeletal muscle nutritional intervention via blended protein supplementation in posttransplant patients with hematological malignancies. Clin Nutr, 2020.

49. Haro, C., O.A. Rangel-Zúñiga, J.F. Alcalá-Díaz, F. Gómez-Delgado, P. Pérez-Martínez, J. Delgado-Lista, et al., Intestinal Microbiota Is Influenced by Gender and Body Mass Index. 2016. 11(5): p. e0154090.

50. Indiani, C., K.F. Rizzardi, P.M. Castelo, L.F.C. Ferraz, M. Darrieux, and T.M. Parisotto, Childhood Obesity and Firmicutes/Bacteroidetes Ratio in the Gut Microbiota: A Systematic Review. Child Obes, 2018. 14(8): p. 501-509.

51. Riva, A., F. Borgo, C. Lassandro, E. Verduci, G. Morace, E. Borghi, et al., Pediatric obesity is associated with an altered gut microbiota and discordant shifts in Firmicutes populations. Environ Microbiol, 2017. 19(1): p. 95-105.

52. Norman, K., N. Stobäus, M.C. Gonzalez, J.D. Schulzke, and M. Pirlich, Hand grip strength: outcome predictor and marker of nutritional status. Clin Nutr, 2011. 30(2): p. 135-42.

53. Alekseev, O.M., R.T. Richardson, O. Alekseev, and M.G. O'Rand, Analysis of gene expression profiles in HeLa cells in response to overexpression or siRNA-mediated depletion of NASP. Reproductive biology and endocrinology : RB\&E, 2009. 7: p. 45-45.

54. Kang, X., Y. Feng, Z. Gan, S. Zeng, X. Guo, X. Chen, et al., NASP antagonize chromatin accessibility through maintaining histone H3K9me1 in hepatocellular carcinoma. Biochim Biophys Acta Mol Basis Dis, 2018. 1864(10): p. 3438-3448.

55. Zhan, Q., J. Zhang, H. Wang, X. Wu, Y. Zhu, Z. Xu, et al., [Mutation of nuclear autoantigenic sperm protein (NASP) gene aggravates autoimmune response in induced lupus model mice]. Xi Bao Yu Fen Zi Mian Yi Xue Za Zhi, 2019. 35(9): p. 776-782.

56. Richardson, R.T., O.M. Alekseev, G. Grossman, E.E. Widgren, R. Thresher, E.J. Wagner, et al., Nuclear autoantigenic sperm protein (NASP), a linker histone chaperone that is required for cell proliferation. J Biol Chem, 2006. 281(30): p. 21526-34.

57. Yang, F.-M., Y. Zuo, W. Zhou, C. Xia, B. Hahm, M. Sullivan, et al., sNASP inhibits TLR signaling to regulate immune response in sepsis. The Journal of clinical investigation, 2018. 128(6): p. 2459-2472.

Page $12 / 13$ 
58. Kumar, A., S. Bhatnagar, and P.K. Paul, TWEAK and TRAF6 regulate skeletal muscle atrophy. Curr Opin Clin Nutr Metab Care, 2012. 15(3): p. 233-9.

59. Qiu, J., J. Zhu, R. Zhang, W. Liang, W. Ma, Q. Zhang, et al., miR-125b-5p targeting TRAF6 relieves skeletal muscle atrophy induced by fasting or denervation. Ann Transl Med, 2019. 7(18): p. 456.

60. Norman, K., N. Stobäus, K. Kulka, and J. Schulzke, Effect of inflammation on handgrip strength in the non-critically ill is independent from age, gender and body composition. European Journal of Clinical Nutrition, 2014. 68(2): p. 155-158.

61. Carter, M.T., H.J. McMillan, A. Tomin, and N. Weiss, Compound heterozygous CACNA1H mutations associated with severe congenital amyotrophy. Channels (Austin, Tex.), 2019. 13(1): p. 153-161.

62. Li, S., M. Hao, B. Li, M. Chen, J. Chen, J. Tang, et al., CACNA1H downregulation induces skeletal muscle atrophy involving endoplasmic reticulum stress activation and autophagy flux blockade. Cell death \& disease, 2020. 11(4): p. 279-279.

\section{Figures}

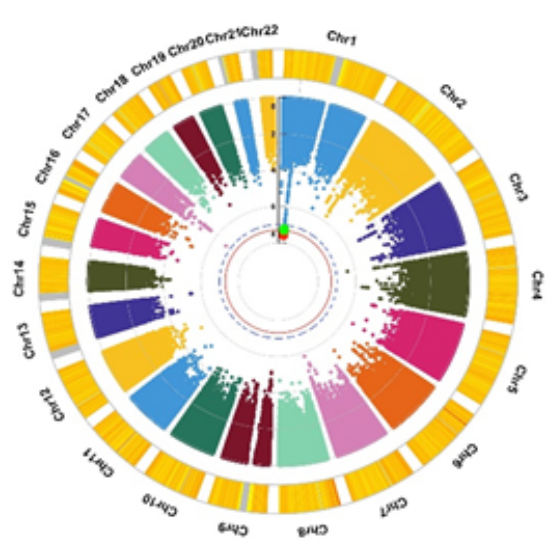

A

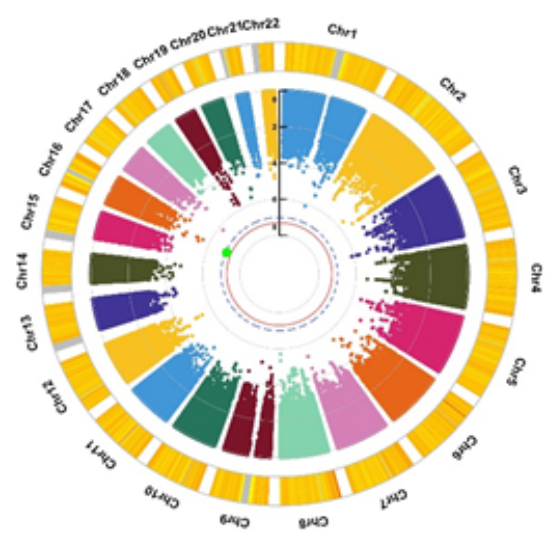

C
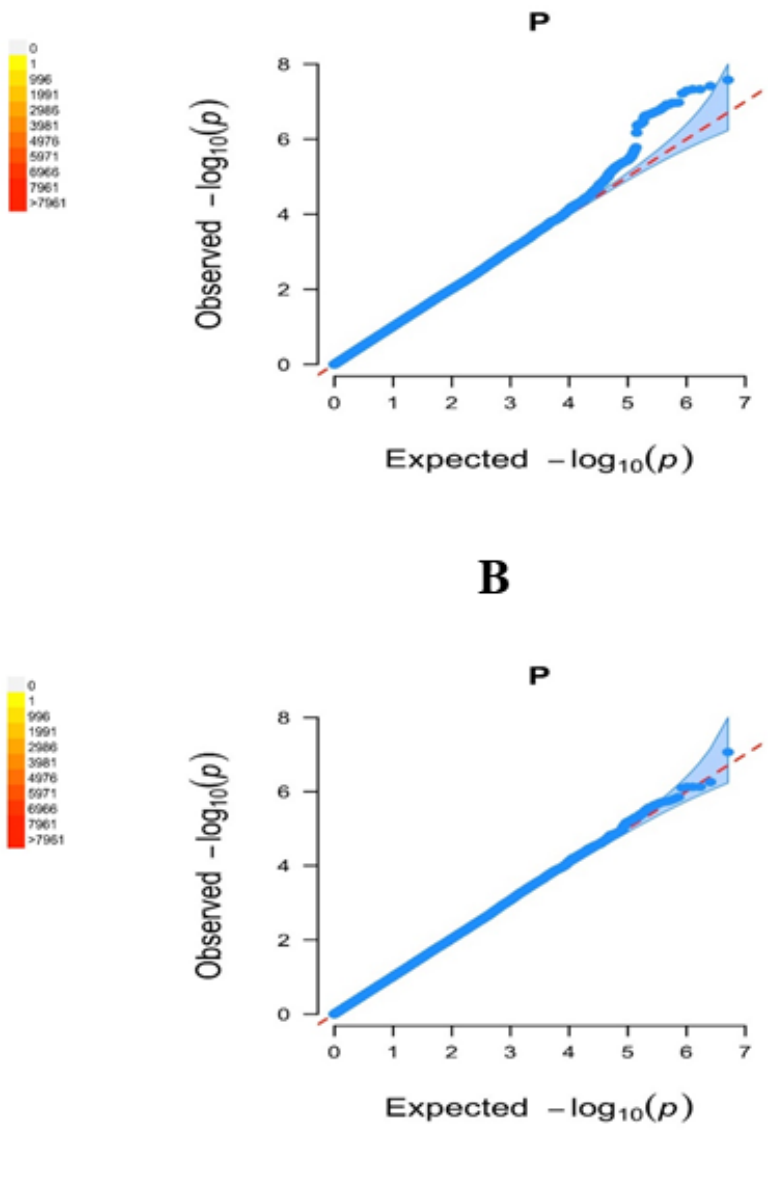

D

\section{Figure 1}

Genome-wide gene-GM interactions study of HGS. A and C: the Manhattan plots for genome-wide gene-G_Butyricicoccus interactions study for right HGS $(P<5 \times 10-8)$ and left HGS $(P<1 \times 10-7)$, respectively. $B$ and $D$ : the QQ plots of right HGS and left HGS, respectively. QQ plot is a graphical representation of the deviation of the observed $\mathrm{P}$ values from the null hypothesis: the observed $\mathrm{P}$ values for each SNP are sorted from largest to smallest and plotted against expected values from a theoretical $\chi 2$-distribution. 